\title{
Downregulation of TRIM27 expression inhibits the proliferation of ovarian cancer cells in vitro and in vivo
}

\author{
Yanyan Ma ${ }^{1,5}$, Zengtao Wei ${ }^{2,3,5}$, Robert C Bast $\mathrm{Jr}^{4}$, Zhanying Wang ${ }^{3}$, Yan Li', Meng Gao ${ }^{1}$, Yanping Liü ${ }^{2,3}$, \\ Xiaoyan Wang ${ }^{1}$, Chun Guo ${ }^{1}$, Lining Zhang ${ }^{1}$ and Xiaoyan Wang ${ }^{1}$
}

TRIM27 (tripartite motif-containing 27) was originally identified as a fusion partner with the RET (REarranged during transfection) proto-oncogene and is highly expressed in various tumor cells and tissues. However, the level of expression and function of TRIM27 in ovarian cancer remain unclear. Here we have measured the expression of TRIM27 in normal ovarian and fallopian tube epithelial cells and in ovarian serous carcinoma cells and correlated TRIM27 expression with clinical and pathological parameters. In addition, we detected the effect of TRIM27 knockdown on proliferation of ovarian cancer cells in cell culture and xenografts. The results demonstrated that TRIM27 was highly expressed in ovarian serous carcinoma cells, and TRIM27 expression was significantly correlated with metastasis and FIGO stage in ovarian serous carcinoma patients. Downregulation of TRIM27 expression suppressed the proliferation of ovarian cancer cells in cell culture and inhibited the growth of xenografts in nude mice. TRIM27 knockdown induced cell cycle arrest and apoptosis in ovarian cancer cells by upregulating the expression of p-P38 and downregulating the expression of p-AKT. Thus the present study suggests that TRIM 27 could have important roles as an oncogene during the development of ovarian cancer and could serve as a diagnostic and therapeutic target.

Laboratory Investigation (2016) 96, 37-48; doi:10.1038/labinvest.2015.132; published online 16 November 2015

Although ovarian cancer occurs less frequently than cervical or endometrial cancer, it is the most lethal of the gynecological malignancies. ${ }^{1}$ Ovarian cancer patients can have symptoms prior to diagnosis, but these symptoms are shared with many more common benign conditions, making early detection difficult. As only $20-25 \%$ are diagnosed in stages I-II, the 5 -year survival rate is still $<30 \% .^{2}$ The etiology and molecular mechanisms contributing to ovarian cancer are still not well known. Understanding the pathogenesis of ovarian cancer at a molecular level may aid in developing prognostic biomarkers and targets for more effective treatment against ovarian cancer.

TRIM27 (tripartite motif-containing 27) belongs to the zinc finger protein superfamily, which contains a tripartite motif consisting of a RING finger, B-box zinc finger and coiled-coil domain., ${ }^{3,4}$ It was originally identified as a fusion partner with the RET (REarranged during transfection) proto-oncogene, which encodes a receptor tyrosine kinase; therefore, it is also known as RFP (RET finger protein).
TRIM27 exhibits oncogenic properties in mouse embryos NIH3T3 cells when its tripartite domain is recombined with the tyrosine kinase domain of the RET proto-oncogene by DNA rearrangement. ${ }^{5}$ It has been reported that TRIM27 is detectable in male germ cells, peripheral and central neurons, hepatocytes and adrenal chromaffin cells. ${ }^{6-8}$ However, it is highly expressed in various tumor cells and tissues, such as endometrial cancer, breast cancer and lung cancer. Furthermore, high-level expression of TRIM27 correlates with the proliferation, migration and chemosensitivity of some tumor cells and could act as a potential prognostic indicator in some cancers. ${ }^{9-12}$ The above results suggest that TRIM27 could be involved in the development and progression of tumor. However, its expression level and function in ovarian cancer remain unclear.

Ovarian cancers are classified according to their microscopic appearance. Among different types, high-grade ovarian serous carcinoma is the most common histological type of ovarian cancers and originates from epithelial cells of the

\footnotetext{
${ }^{1}$ Department of Immunology, Shandong University School of Medicine, Jinan, Shandong, P. R. China; ${ }^{2}$ Department of Gynecology, Shandong University School of Medicine, Jinan, Shandong, P. R. China; ${ }^{3}$ Department of Gynecology, Jinan Central Hospital Affiliated to Shandong University, Jinan, Shandong, P. R. China and ${ }^{4}$ Department of Experimental Therapeutics, Division of Cancer Medicine, The University of Texas MD Anderson Cancer Center, Houston, TX, USA

Correspondence: Dr X Wang, Associate Professor of Immunology, Department of Immunology, Shandong University School of Medicine, 44\# Wenhua Xi Road, Jinan, Shandong 250012, P. R. China.

E-mail: wxy990809@163.com or wxy1970@sdu.edu.cn

${ }^{5}$ These authors contributed equally to this work.
}

Received 21 January 2015; revised 22 August 2015; accepted 26 August 2015 
Table 1 Relationship of TRIM27 expression with clinical and pathological parameters in ovarian serous carcinoma patients $(* P<0.05)$

\begin{tabular}{lcc}
\hline Clinical and \\
pathological features
\end{tabular}

$\begin{array}{lrrrr}\begin{array}{l}\text { Age, years } \\ \leq 55\end{array} & 26 & 12 & 14 & P=0.2597 \\ >55 & 24 & 7 & 16 & \end{array}$

Site of origin

$\begin{array}{lllll}\text { Double } & 22 & 8 & 14 & P=0.7593 \\ \text { Single } & 17 & 7 & 10 & \end{array}$

Metastasis

$\begin{array}{lcccc}\text { Positive } & 34 & 11 & 23 & * \\ \begin{array}{l}\text { Fallopian tube or } \\ \text { uterus metastasis }\end{array} & 31 & & \\ \begin{array}{l}\text { Omentum or } \\ \text { intestinal wall or }\end{array} & 26 & \\ \text { appendix metastasis } & \\ \text { Lymph node } & 6 & \\ \text { metastasis } & & \\ \text { Distant metastasis } & 3 & & \\ \text { Negative } & 12 & 8 & 4\end{array}$

Pathological grade

$\begin{array}{lrrrr}\text { Low differentiation } & 32 & 9 & 23 & P=0.0801 \\ \text { Middle-high } & 18 & 10 & 9 & \end{array}$

differentiation

\begin{tabular}{lrrrr} 
FIGO stage & & & & \\
I-II & 15 & 9 & 6 & $* P=0.0445$ \\
III-IV & 28 & 8 & 20 & \\
\hline
\end{tabular}

ovary and fallopian tube. In the current study, we have measured the expression of TRIM27 in normal epithelial cells of the ovary and fallopian tube and in ovarian serous carcinoma cells. We have correlated TRIM27 expression with clinical and pathological characteristics of patients with ovarian serous carcinoma. In addition, we detected the effect of TRIM27 knockdown on proliferation of ovarian serous carcinoma cells in cell cultures and xenografts. The results demonstrated that TRIM27 was highly expressed in ovarian serous carcinoma cells, and TRIM27 expression was significantly correlated with metastasis and International Federation of Gynecology and Obstetrics (FIGO) stage. Downregulation of TRIM27 expression not only suppressed the proliferation of ovarian cancer cells in cell culture but also inhibited the growth of xenografts in nude mice. Therefore, the present study suggests that TRIM27 may be an important target for prognostication and possibly for treatment of ovarian cancer.

\section{MATERIALS AND METHODS Patients and Tissue Samples}

Fifty ovarian serous carcinoma patients aged 30-79 years (the average age is 50 years) were recruited from Jinan Central Hospital affiliated to Shandong University from 2001 to 2012. None of them had received radiotherapy or chemotherapy prior to surgery. Tumor samples were graded based on Gynecologic Oncology Group Criteria and staged in accordance with the FIGO system. Twenty-six normal controls, including 16 normal ovaries and 10 normal fallopian tubes, were acquired from patients without cancer aged between 35 and 65 years who had undergone surgical removal of the ovaries or fallopian tubes. The study protocol was approved by the ethical committee affiliated to Jinan Central Hospital of Shandong University, and informed consent was obtained from all the patients and controls. The characteristics of the patients are summarized in Table 1 .

\section{Antibodies}

Rabbit polyclonal antibody specific for TRIM27 was purchased from Proteintech Group (Wuhan, Hubei, China). Rabbit polyclonal antibodies specific for ERK1/2, p-ERK1/2, SAPK/JNK, p-SAPK/JNK, p38 MAPK, p-p38 MAPK, AKT, p-AKT, ATR (Ataxia Telangiectasia and Rad3 related) and p-ATR were purchased from Cell Signaling Technology (Boston, MA, USA). Mouse monoclonal antibody specific for Chk1 (checkpoint kinase 1) and rabbit monoclonal antibody specific for p-Chk1 were also purchased from Cell Signaling Technology. Rabbit polyclonal antibody specific for $\beta$-actin was purchased from ImmunoWay (Newark, DE, USA).

\section{Immunohistochemistry (IHC) Analysis}

TRIM27 expression in 50 ovarian serous carcinomas and 26 normal control specimens was detected by IHC using the Histostain-Plus Kit (Beijing 4A Biotech, Beijing, China). The formalin-fixed and paraffin-embedded tumor tissue sections $(4 \mu \mathrm{m})$ were deparaffinized in xylene and rehydrated in graded ethanol, followed by antigen microwave retrieval. The slides were washed and blocked with $3 \% \mathrm{H}_{2} \mathrm{O}_{2}$ and $10 \%$ goat serum and then incubated with anti-TRIM27 (1:100) overnight at $4{ }^{\circ} \mathrm{C}$. Next the slides were incubated with HRP-conjugated anti-rabbit IgG and Streptavidin/HRP, followed by diaminobenzidine (DAB) staining. The nuclei were counterstained with hematoxylin. All IHC staining was independently assessed by two experienced pathologists. The nuclear and cytoplasmic TRIM27 staining was divided into four grades according to staining intensity: - (score 0), $+($ score 1$),++$ (score 2$)$, and +++ (score 3$)$. The percentage 
Table 2 Sequences of siRNA used for TRIM27 knockdown

\begin{tabular}{ll}
\hline Name & Sequence \\
\hline TRIM27-homo-918 & 5'-GCAGCUGUAUCACUCCUUATT-3' \\
T'-UAAGGAGUGAUACAGCUGCTT-3' & 5'-GCAGUCAGAUAUGGACAAATT-3' \\
TRIM27-homo-1251 & 5'-UUUCUCCAUAUCUGACUGCTT-3' \\
& 5'-GGCAGUGUCUUUGUGGUAUTT-3' \\
TRIM27-homo-1578 & 5'-AUACCACAAAGACACUGCCTT-3' \\
& 5'-GUAUGACAACAGCCUCAAGTT-3' \\
GAPDH-positive control & 5'-CUUGAGGCUGUUGUCAUACTT-3' \\
& 5'-UUCUCCGAACGUGUCACGUTT-3' \\
GAPDH-negative control & 5'-ACGUGACACGUUCGGAGAATT-3'
\end{tabular}

of TRIM27-positive cells was also classified into four categories: - $(<1 \%$, score 0$),+(1-33 \%$, score 1$)$, $++(34-66 \%$, score 2$)$, and $+++(67-100 \%$, score 3$)$. The sum of intensity and percentage scores was used as the final TRIM27 staining scores, defined as follows: no expression (total score 0 ); weak expression (total score 1 and 2); moderate expression (total score 3 and 4); and strong expression (total score 5 and 6$)$.

\section{Cell Culture}

Human ovarian serous carcinoma-derived cells SKOV3, OVCAR3 and CAOV3 were purchased form China Center for Type Culture Collection (Wuhan), and human ovarian mucinous carcinoma-derived cells $3 \mathrm{AO}$ were purchased from Shandong Academy of Medical Sciences (Jinan, Shandong, China). These cells were incubated at $37^{\circ} \mathrm{C}$ in a humidified atmosphere of $5 \% \mathrm{CO}_{2}$. SKOV3 cells were cultured in McCoy's 5a (Gibco, CA, USA) containing 10\% fetal bovine serum (FBS) (Gibco). CAOV3 cells were cultured and maintained in Dulbecco's modified Eagle's medium (Hyclone, Utah, USA) containing 10\% FBS. OVCAR3 cells were cultured in RPMI-1640 medium (Gibco), supplemented with $20 \% \mathrm{FBS}$ and $1 \mathrm{mM}$ sodium pyruvate and $2 \mathrm{mM}$ L-glutamine. $3 \mathrm{AO}$ cells were cultured in RPMI 1640 medium (Hyclone) supplemented with $10 \%$ FBS. The above culture media contain penicillin $(100 \mathrm{U} / \mathrm{ml})$ and streptomycin $(100 \mu \mathrm{g} / \mathrm{ml})$.

\section{siRNA and Transfection}

SKOV3 and OVCAR3 cells were transfected with siRNA targeting TRIM27 (GenePharma, Shanghai, China) and negative control siRNA using Lipofectamine 2000 according to the manufacturer's protocols (Invitrogen, Carlsbad, CA, USA). The target sequences are shown in Table 2.

\section{RNA Isolation and RT-PCR}

Total RNA was extracted from ovarian cancer cells with TRIZOL regent (Invitrogen). cDNA was synthesized using the
Reverse- Transcribe Kit (TIANGEN, Beijing, China) according to the manufacturer's instructions. RT-PCR was performed with TRIM27-specific primers (sense 5'-AGCAA

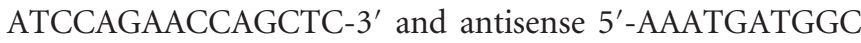
ACCATTGATG- $3^{\prime}$ ) and $2 \times$ Taq MasterMix (TIANGEN) for 33 cycles $\left(95^{\circ} \mathrm{C}\right.$ for $30 \mathrm{~s}, 58^{\circ} \mathrm{C}$ for $30 \mathrm{~s}$ and $72{ }^{\circ} \mathrm{C}$ for $\left.30 \mathrm{~s}\right)$. Human $\beta$-actin (sence $5^{\prime}$-AGGCCAACCGCGAGAAGATG-3' and antisense $5^{\prime}$-CACACGGAGTACTTGCGCTCAG-3') were amplified as internal control.

\section{Western Blotting}

Cells were washed in PBS and collected in RIPA lysis buffer (Beyotime, Shanghai, China). Equal amount of protein was separated on SDS-polyacrylamide gel and then electrotransferred to PVDF membranes (Millipore, Billerica, MA, USA). The membranes were blocked with $2 \%$ BSA for $1 \mathrm{~h}$ at room temperature followed by incubation with rabbit anti-human TRIM27 antibody (1:1000), rabbit or mouse anti-human ERK1/2, p-ERK1/2, SAPK/JNK, p-SAPK/JNK, p38 MAPK, p-p38 MAPK, AKT, p-AKT, ATR, p-ATR, Chk1 and p-Chk1 (1:1000) overnight at $4{ }^{\circ} \mathrm{C}$ and then incubated with 1:2000 dilution of secondary antibodies (goat anti-rabbit or mouse IgG). After washing, the signals were detected by the ECL Western Blot Kit (Thermo Scientific, MA, USA). The intensity of the target protein bands was normalized to the loading control $\beta$-actin.

\section{Cell Viability Assay}

SKOV3 and OVCAR3 cells were, respectively, transfected with siRNA targeting TRIM27 and negative control siRNA using Lipofectamine 2000. Twenty-four hours later, the cells were reseeded in 96-well plates at 3000 cells/well, and the cells were allowed to grow for 24, 48 and $72 \mathrm{~h}$. Cell viability was evaluated using CCK8 (Dojindo, Kumamoto, Japan) according to the manufacturer's instructions. The absorbance was determined at $450 \mathrm{~nm}$ using Bio-Rad Microplate Reader. The experiments were performed in triplicate and repeated three times.

\section{Colony-Formation Assay}

SKOV3 or OVCAR3 cells were transfected with siRNA targeting TRIM27 and negative control siRNA. Twenty-four hours later, the cells were reseeded in six-well plates at 250 cells/well for SKOV3 or 500 cells/well for OVCAR3 cells. Two or 3 weeks later, the colonies were fixed in methanol for $20 \mathrm{~min}$ and then stained with $0.1 \%$ crystal violet. The number of colonies with $\geq 50$ cells was counted, and colony forming efficiency was calculated (percentage of colonies $=$ number of colonies formed/number of cells inoculated $\times 100 \%$ ). The experiments were performed in duplicate and repeated three times.

\section{Cell Cycle Analysis}

SKOV3 cells were transfected with siRNA targeting TRIM27 and negative control siRNA. After $48 \mathrm{~h}$, the cells were washed 

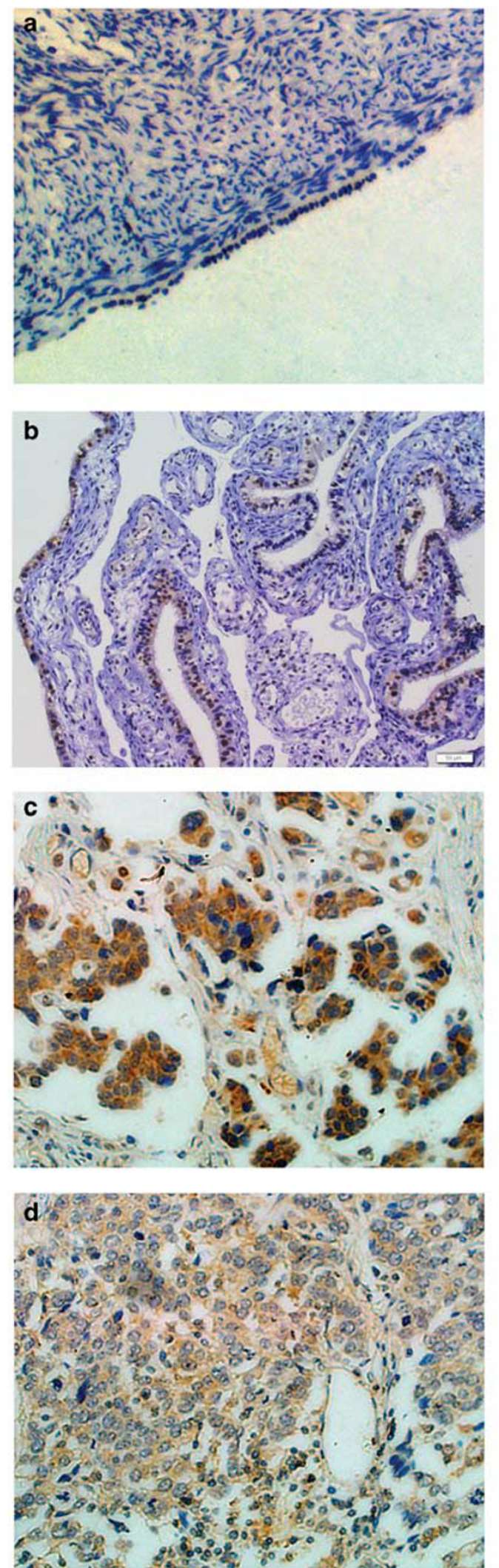
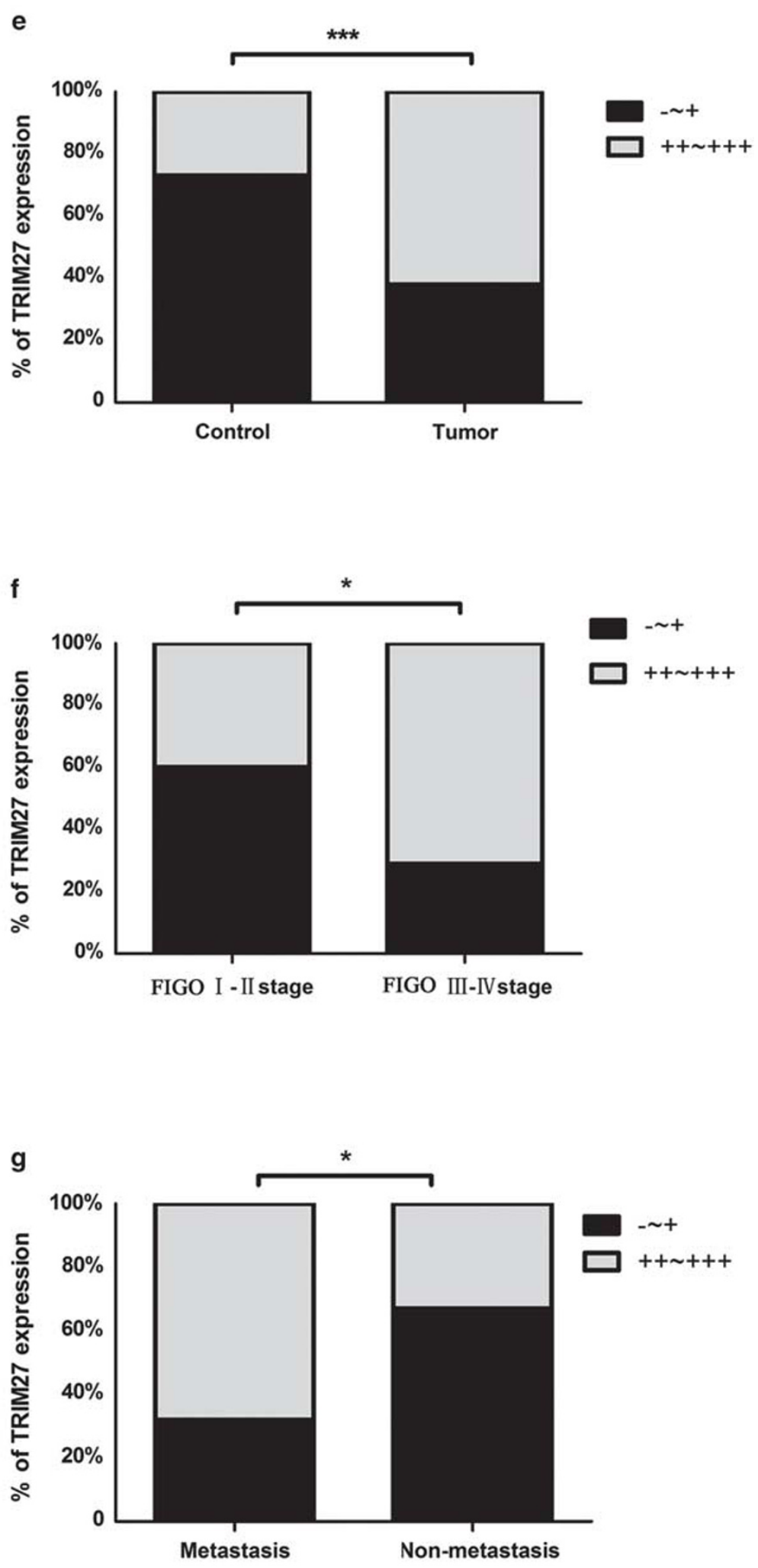
with cold PBS and fixed with 70\% ethanol. The fixed cells were resuspended in PBS at a concentration of $1 \times 10^{6}$ cells $/ \mathrm{ml}$ and incubated with $100 \mu \mathrm{g} / \mathrm{ml}$ of RNaseA at $37^{\circ} \mathrm{C}$ for $30 \mathrm{~min}$. The cell suspension was stained by propidium iodide (PI), and DNA content was evaluated by flow cytometry using Beckman Coulter Cytomics FC500 (Beckman Coulter, Fullerton, CA, USA). The experiment was repeated three times.

\section{Cell Apoptosis Analysis}

Cell apoptosis was assessed using the Annexin V and PI Kit (Dojindo) according to the manufacturer's instructions. Briefly, SKOV3 cells were transfected with siRNA targeting TRIM27 and negative control siRNA. After $48 \mathrm{~h}$, the cells were collected and washed twice with cold PBS. Then the cells were resuspended in binding buffer at a concentration of $1 \times 10^{6} \mathrm{cells} / \mathrm{ml}$. One hundred microliters of the cell suspension $\left(1 \times 10^{5}\right.$ cells $)$ was transferred to a $5-\mathrm{ml}$ culture tube. Next, $5 \mu \mathrm{l}$ of annexin V-APC and $5 \mu \mathrm{l}$ of PI were added to the cell suspension and incubated for $15 \mathrm{~min}$ at room temperature in the dark. The stained cells were then diluted to $500 \mu$ l with binding buffer and analyzed with flow cytometry using the Beckman Coulter Cytomics FC500 (Beckman Coulter). The experiment was repeated three times.

\section{In Vivo Study Using a Xenograft Nude Mouse Model}

Fifteen female nude mice (Vital River Laboratories, Beijing, China) at 6-8 weeks of age were inoculated subcutaneously into the left flank with $9 \times 10^{6}$ SKOV3 cells in $100 \mu$ normal saline (NS). Two weeks later, these mice were randomly divided into three groups, five mice/group. They were

Table 3 Statistical analysis of TRIM27 expression in normal ovarian and fallopian tube tissues and in ovarian serous carcinomas $(* * P<0.001)$

\begin{tabular}{|c|c|c|c|c|c|c|}
\hline & \multirow[t]{2}{*}{$n$} & \multicolumn{4}{|c|}{$\begin{array}{l}\text { TRIM27 } \\
\text { expression }\end{array}$} & \multirow[t]{2}{*}{$P$-value } \\
\hline & & - & + & ++ & +++ & \\
\hline Ovarian serous carcinomas & 50 & 9 & 10 & 22 & 9 & ${ }^{* * *} P<0.001$ \\
\hline $\begin{array}{l}\text { Normal ovarian and fallopian tube } \\
\text { tissues }\end{array}$ & 26 & 7 & 12 & 4 & 3 & \\
\hline
\end{tabular}

respectively treated with $30 \mu \mathrm{g}$ of control shRNA (sense $5^{\prime}$-TT CTCCGAACGTGTCACGT- $3^{\prime}$ and antisense $5^{\prime}$-ACGTGAC ACGTTCGGAGAA-3') or TRIM27 shRNA (sense 5'-GGCAG TGTCTTTGTGGTATGGT- $3^{\prime}$ and antisense 5'-ACCATACC ACAAAGACACTGCC-3') (Sangon Botech, Shanghai, China) in $100 \mu \mathrm{l}$ NS by intra-tumor injection every other day, for a total of five times injections. Intra-tumor injection of $100 \mu \mathrm{l}$ NS acted as the control group. Tumor volume was assessed every day and calculated as follows: length $\times$ width $\times$ width $\times 0.4$. All mice were killed after completion of treatment and tumors were removed for measurement of volume and weight. In addition, western blots were used to detect the efficiency of TRIM27 knockdown in tumors. The protocol was approved by the Animal Care and Utilization Committee of Shandong University. This study was also performed in full compliance with the guidelines for the welfare of animals in experimental neoplasia.

\section{Statistical Analysis}

The results were expressed as mean \pm s.e.m. The Chi-square test and Student's $t$-test were performed to evaluate the statistical significance between two groups. $P<0.05$ was considered to indicate a statistically significant difference.

\section{RESULTS}

TRIM27 Expression Was Increased in Ovarian Serous Carcinoma Cells and Correlated Positively to FIGO Stage And Tumor Metastasis

IHC results from ovarian serous carcinoma cells showed that about $18 \%(9 / 50)$ were negative, $20 \%(10 / 50)$ expressed low levels of TRIM27, 44\% (22/50) expressed medium levels of TRIM27 and 18\% (9/50) expressed high levels of TRIM27. The positive staining for TRIM27 mainly existed in the cytoplasma of ovarian serous carcinoma cells, only a few of carcinoma cells expressed TRIM27 in the cytoplsma and nucleus. On the other hand, in normal ovarian and fallopian tube epithelial cells, $26.9 \%$ (7/26) were negative, $46.2 \%$ (12/26) expressed low levels of TRIM27, only $15.4 \%(4 / 26)$ expressed medium levels of TRIM27 and $11.5 \%$ (3/26) expressed high levels of TRIM27 (Figures 1a-d,Table 3). The TRIM27 staining was located in the cytoplasma of normal ovarian and fallopian tube epithelial cells. By statistical analysis, the expression of TRIM27 protein in ovarian serous carcinoma cells was significantly higher than that in normal controls (Figures 1 e, $P<0.001$ ). We further analyzed the relationships between the TRIM27

Figure 1 TRIM27 expression was increased in ovarian serous carcinoma cells and correlated positively to FIGO stage and tumor metastasis. (a) Expression of TRIM27 in normal ovarian tissues was detected by immunohistochemistry; the normal ovarian epithelial cells showed negative or low level of TRIM27 staining. (b) Expression of TRIM27 in normal fallopian tube tissues was detected by immunohistochemistry; the normal fallopian tube epithelial cells showed negative or low level of TRIM27 staining. (c and d) Expression of TRIM27 in ovarian serous carcinoma tissues was detected by immunohistochemistry; the ovarian serous carcinoma cells showed strong or moderate staining of TRIM27. (e) Expression of TRIM27 protein in ovarian serous carcinoma was significantly higher than that in normal controls $(P<0.001)$. (f) Significantly positive correlation was found between TRIM 27 expression and FIGO stage $(P=0.0445)$ in ovarian serous carcinoma patients by Chi-square test. $(\mathbf{g})$ There was a significantly positive correlation between TRIM27 expression and tumor metastasis $(P=0.0379)$ in ovarian serous carcinoma patients. Data shown are expressed as means \pm s.e.m. ${ }^{*} P<0.05 ;{ }^{* * *} P<0.001$. 


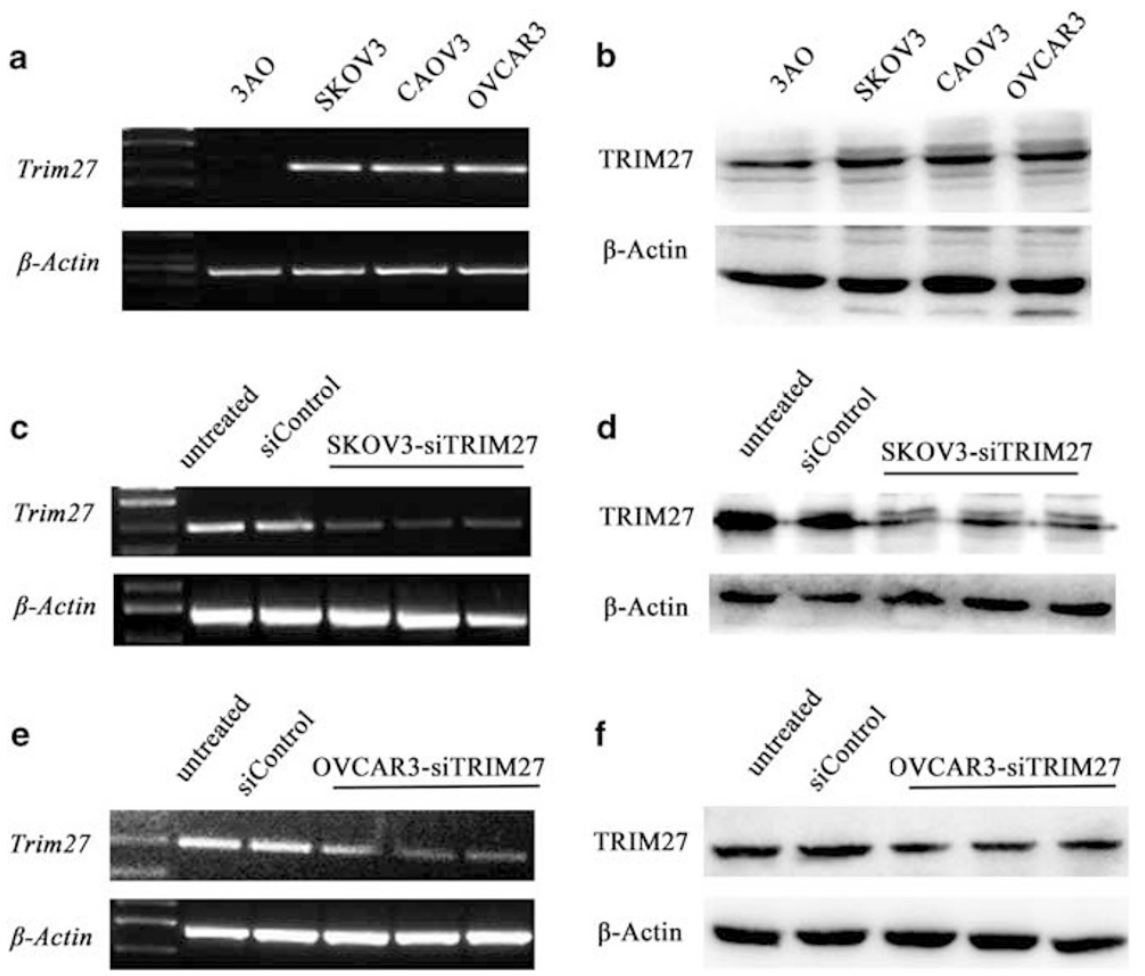

Figure 2 TRIM27 was highly expressed in human ovarian serous carcinoma-derived cell lines and TRIM27-specific siRNA downregulated the expression of TRIM27. (a) RT-PCR assay showed that the expression of TRIM27 in human ovarian serous carcinoma-derived SKOV3, OVCAR3 and CAOV3 cells was higher than that in human ovarian mucous carcinoma-derived 3AO cell line. (b) Western blotting assay showed that the expression of TRIM27 in SKOV3, OVCAR3 and CAOV3 cells was higher than that in 3AO cell line. (c and d) SKOV3 cells that were transfected with three different sequences of TRIM27specific siRNA showed lower expression of TRIM27 than the untreated or negative control groups as detected by RT-PCR and western blotting analysis. (e and $\mathbf{f}$ ) OVCAR3 cells that were transfected with three different sequences of TRIM27-specific siRNA showed lower expression of TRIM27 than the untreated or negative control groups as detected by RT-PCR and western blotting analysis.

expression and clinical and pathological parameters of ovarian serous carcinoma patients listed in Table 1 . The results showed that there were significantly positive associations of TRIM27 expression with FIGO stage (Figure 1f, $P=0.0445$ ) and tumor metastasis (Figure 1g, $P=0.0379$ ) in ovarian serous carcinoma patients. However, there was no significant correlation of TRIM27 expression with any other factors, such as age, origin sites and pathological grade. FIGO stage was related to tumor size and metastasis, which suggests that TRIM27 could be involved in tumor growth and migration.

\section{TRIM27 Was Highly Expressed in Human Ovarian Serous Carcinoma-Derived Cell Lines and TRIM27-Specific siRNA Downregulated the Expression of TRIM27}

The expression of TRIM27 in human ovarian serous carcinoma-derived SKOV3, CAOV3, OVCAR3 and human ovarian mucous carcinoma-derived $3 \mathrm{AO}$ cells was detected by RT-PCR and western blotting. The results showed that TRIM27 was generally expressed in these four cell lines, but the expression of TRIM27 in SKOV3, OVCAR3 and CAOV3 cells was higher than that in the $3 \mathrm{AO}$ cell line (Figures $2 \mathrm{a}$ and b). We then transfected SKOV3 and OVCAR3 cells with TRIM27-specific siRNA or negative control siRNA to observe the efficiency of RNA interference. As shown in Figures 2c-f,
SKOV3 and OVCAR3 cells transfected with three different sequences of TRIM27-specific siRNA showed lower expression of TRIM27 than the untreated or negative control groups as detected by RT-PCR and western blotting analysis.

\section{Downregulation of TRIM27 Expression Suppressed the Proliferation and Colony-Forming Ability of Ovarian Cancer Cells}

To determine the effect of TRIM27 on the proliferation of ovarian cancer cells, a CCK8 assay was used to detect cell viability of SKOV3 and OVCAR3 cells after downregulation of TRIM27 expression. The result showed that the SKOV3 cells transfected with TRIM27-specific siRNA grew significantly more slowly than untreated cells or the cells transfected with negative control siRNA (Figures $3 \mathrm{a}, P<0.01$ ). Additionally, the results from colony-formation assay indicated that the colony number and size of SKOV3-siTRIM27 cells were significantly reduced compared with the control groups (Figure $3 \mathrm{c}, P<0.001$ ). The results were further confirmed in OVCAR 3 cells; the cell viability and colony-forming capacity were lower in OVCAR3 cells transfected with TRIM27-specific siRNA than in control cells (Figure $3 \mathrm{~b}, P<0.05$; Figure $3 \mathrm{~d}$, $P<0.01)$. Thus downregulation of TRIM27 expression could 
a
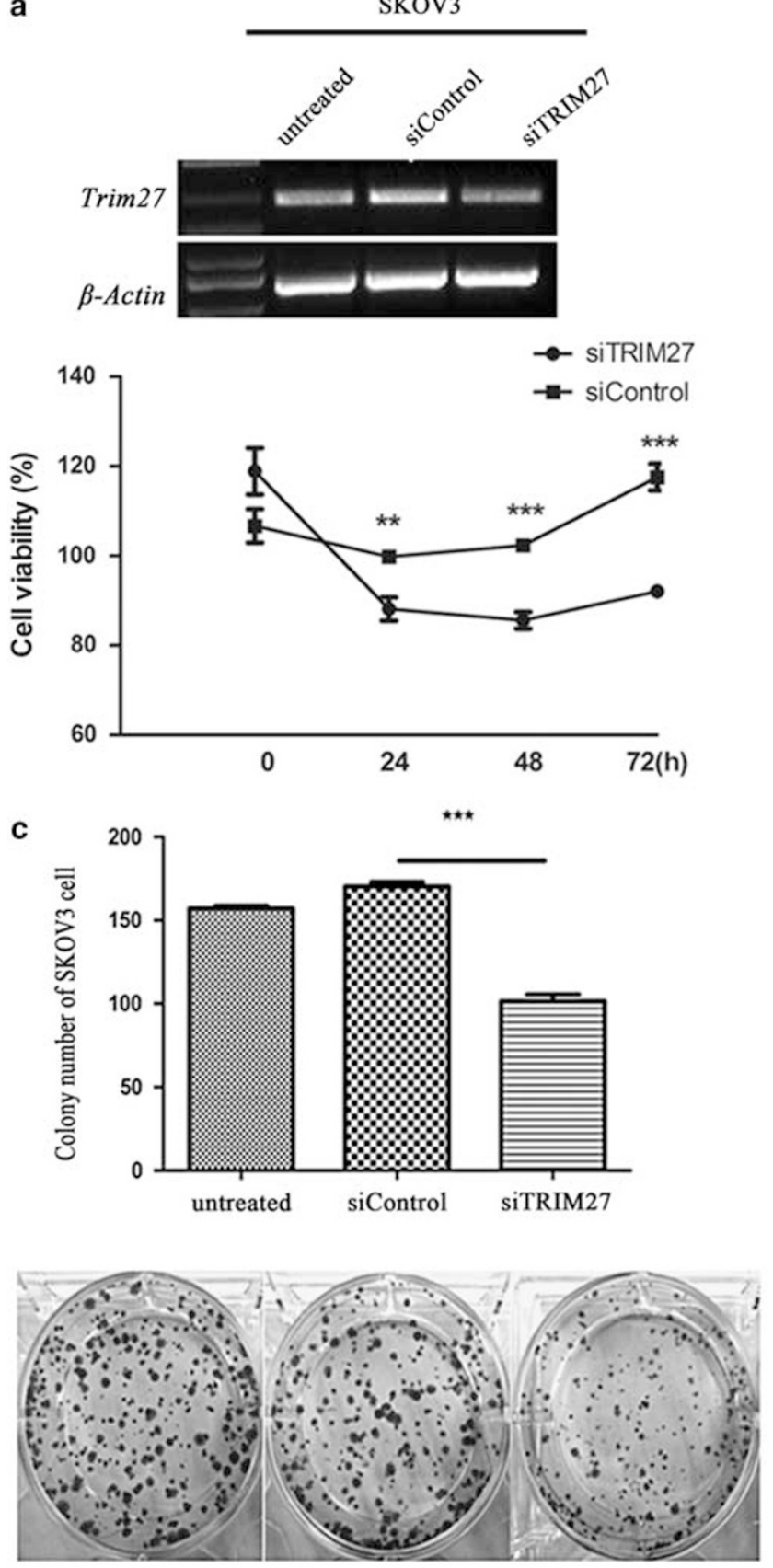

untreated

siControl

siTRIM27

Figure 3 Downregulation of TRIM27 expression suppressed the proliferation and colony-forming ability of ovarian cancer cells. (a and $\mathbf{b})$ SKOV3 and OVCAR3 cells transfected with control and TRIM27 siRNA were used for CCK8 assay. Cell viability in TRIM27 siRNA-transfected cells was significantly reduced compared with corresponding controls. (c and $\mathbf{d}$ ) Reduced expression of TRIM27 in SKOV3 and OVCAR3 cells decreased their colony-forming capacity compared with corresponding controls. ${ }^{*} P<0.05 ;{ }^{*} P<0.01 ; * * P<0.001$.

inhibit the proliferation and colony-forming ability of ovarian cancer cells.

\section{Downregulation of TRIM27 Expression Induced Cell Cycle Arrest and Apoptosis of Ovarian Cancer Cells}

To explore the mechanism(s) by which TRIM27 affects proliferation of ovarian cancer cells, we used flow cytometry b

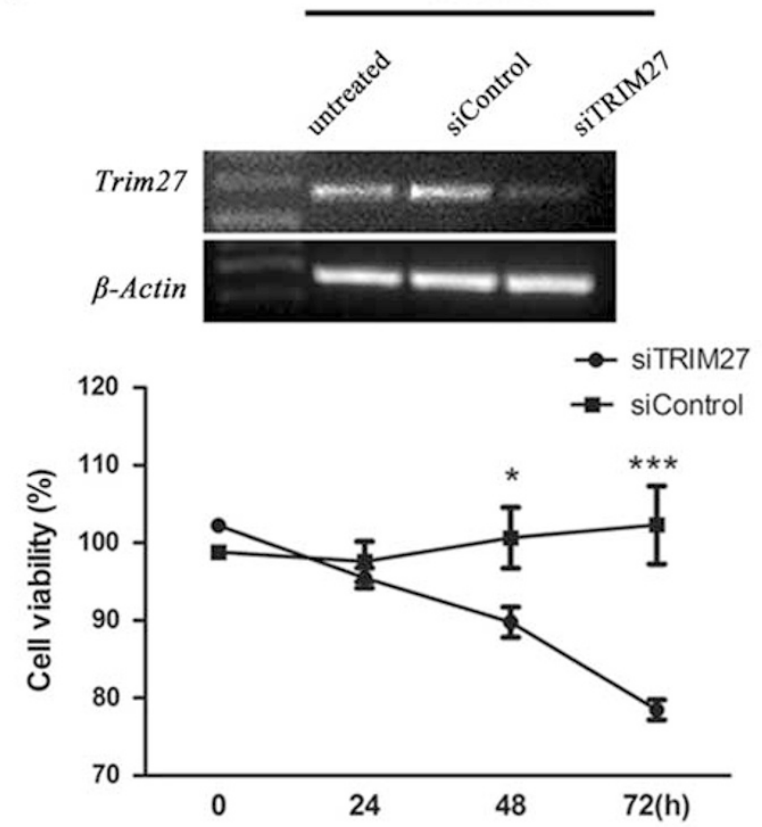

d
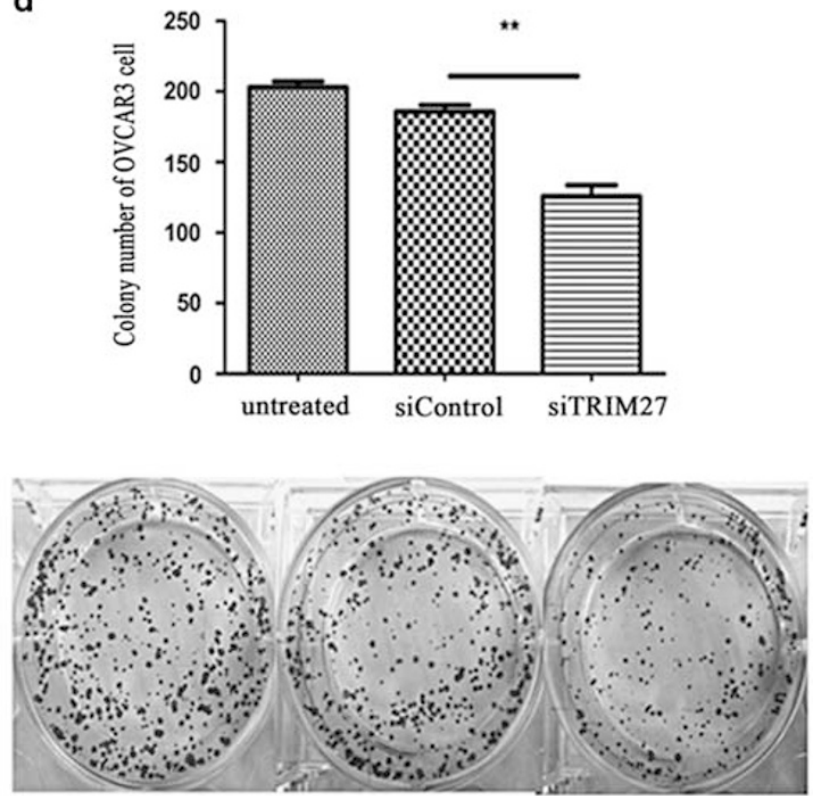

untreated

siControl 

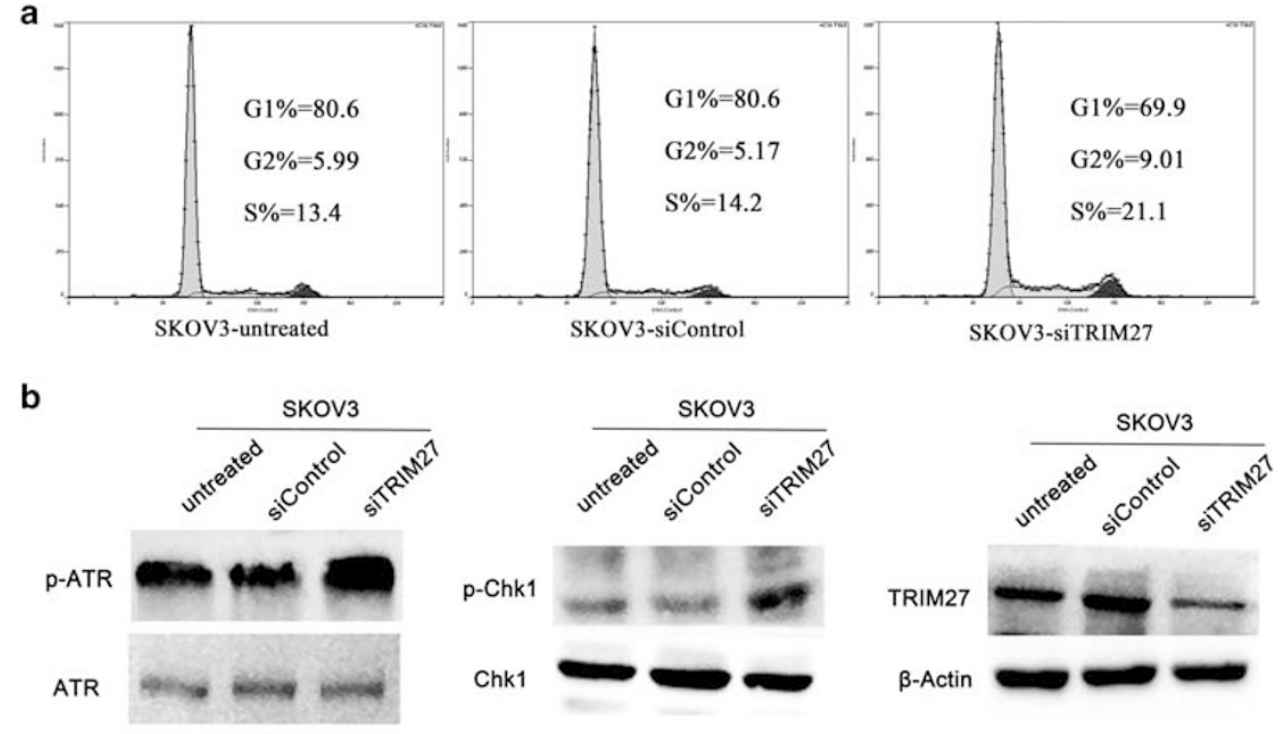

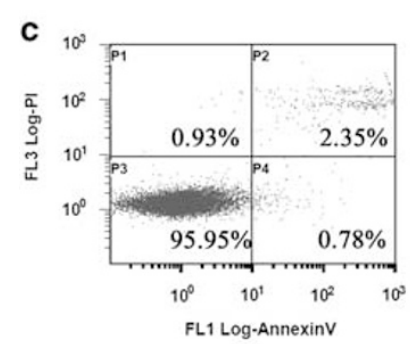

SKOV3-untreated

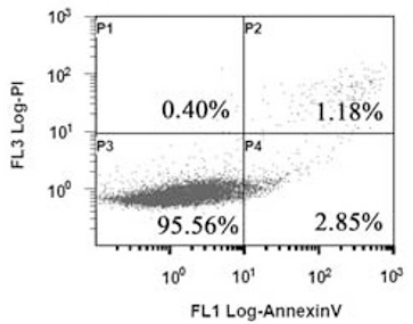

SKOV3-siControl

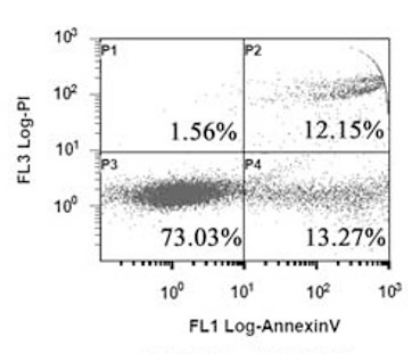

SKOV3-siTRIM27

Figure 4 Downregulation of TRIM27 expression induced cell cycle arrest and apoptosis of ovarian cancer cells. (a) Representative image showed that TRIM27 downregulation in SKOV3 cells delayed cell cycle progression; cell number in S-phase in the siTRIM27 group was markedly increased compared with the siControl and untreated groups $(P<0.05)$. (b) The downregulation of TRIM27 expression increased the expression of p-ATR and p-Chk1.

(c) Representative image showed that reduced TRIM27 expression in SKOV3 cells resulted in a significantly higher apoptosis rate of SKOV3 cells $(P<0.05)$.

expression of p-ATR and p-Chk1 (Figure 4b). In addition, we found that reduced TRIM27 expression resulted in significantly higher apoptosis rate of SKOV3 cells (Figure 4c, $P<0.05)$. These results indicate that downregulation of TRIM27 expression could induce cell cycle arrest and apoptosis of ovarian cancer cells.

\section{Downregulation of TRIM27 Significantly Inhibited the Growth of Ovarian Cancer Cells In Vivo}

To investigate the effect of TRIM27 expression on the growth of ovarian cancer cells in vivo, we established a xenograft nude mouse model using SKOV3 cells. As shown in Figures $5 \mathrm{a}$ and $\mathrm{b}$, the tumors in mice treated with NS or control shRNA grew rapidly, whereas the growth of tumor in mice treated with TRIM27 shRNA was significantly inhibited. After completion of treatment, tumors were removed and tumor volume and weight were measured. The tumor volume in the shTRIM27 group $\left(3.764 \pm 1.161 \mathrm{~mm}^{3}\right)$ was markedly decreased compared with that in the shControl $\left(7.582 \pm 0.9224 \mathrm{~mm}^{3}\right)$ and NS groups $\left(8.922 \pm 1.323 \mathrm{~mm}^{3}\right.$ ) (Figure $5 \mathrm{c}, P<0.05$ ). The tumor weight in the shTRIM27 group $(1.852 \pm 0.557 \mathrm{~g})$ was significantly lower than that in the shControl group $(3.385 \pm 0.273 \mathrm{~g})$ as well as in the NS group $(3.925 \pm 0.678 \mathrm{~g})$ (Figure $5 \mathrm{~d}, P<0.05)$. Furthermore, results of western blotting showed that tumors injected with TRIM27 shRNA expressed lower levels of TRIM27 compared with the shControl and NS groups (Figure 5e). These data suggest that downregulation of TRIM27 expression could inhibit the growth of ovarian cancer cells in vivo.

\section{TRIM27 Played Important Roles by Affecting Cell Proliferation and Apoptosis-Related Pathways}

To determine how TRIM27 has important roles in the cell proliferation and apoptosis, we examined cell proliferation and apoptosis-related pathways (MAPK pathway and AKT pathway) by western blotting. The results showed that downregulated TRIM27 resulted in increased expression of p-P38 in SKOV3 and OVCAR3 cells, while the expression of p-ERK1/2 and p-SAPK/JNK did not change (Figures $6 \mathrm{a}$ and b). In addition, downregulated TRIM27 suppressed the expression of p-AKT in SKOV3 and OVCAR3 cells (Figures $6 \mathrm{c}$ and d). These results indicate that TRIM27 could have important roles by affecting cell proliferation and apoptosis-related pathways. 

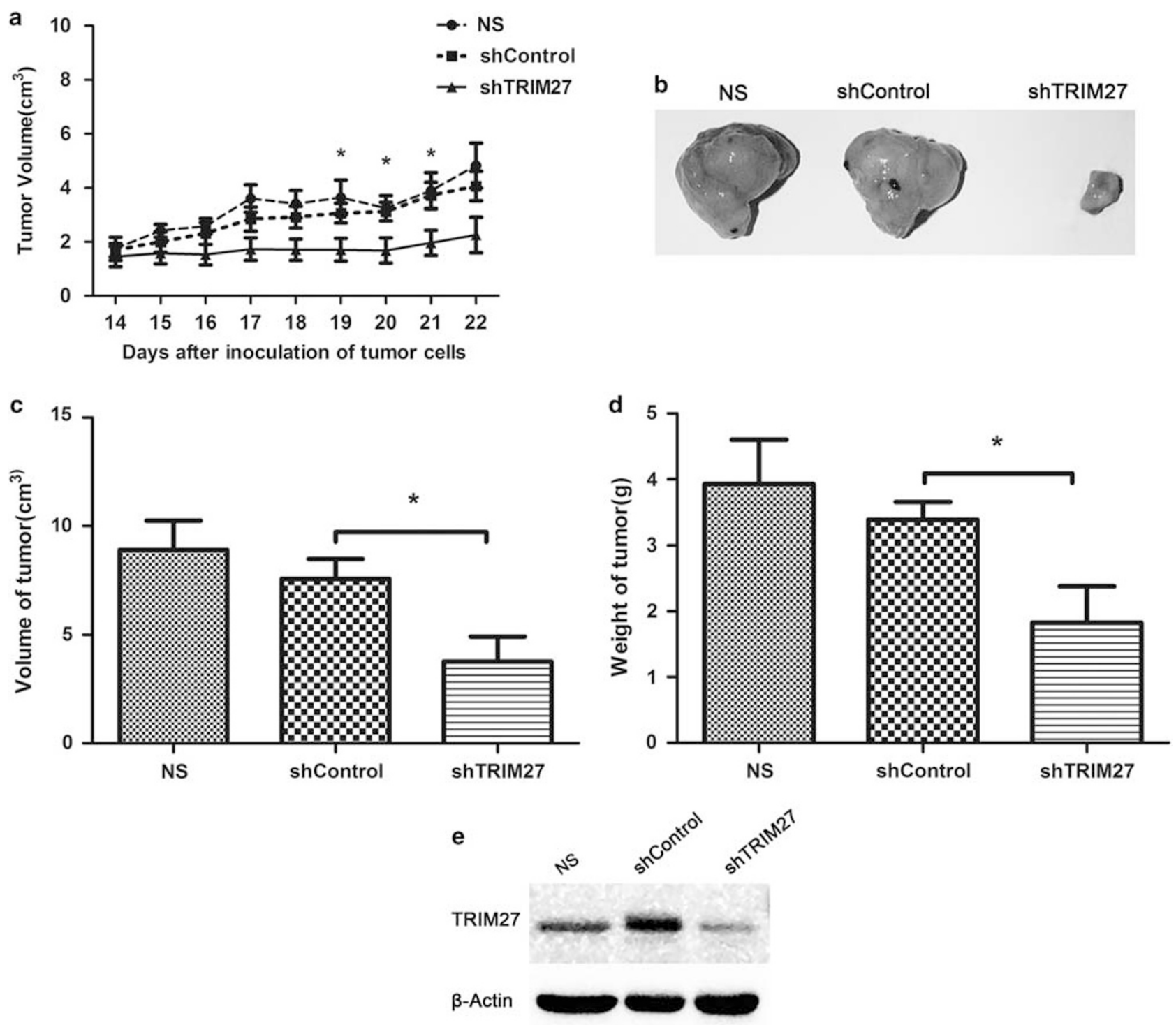

Figure 5 Downregulation of TRIM27 significantly inhibited the growth of ovarian cancer cells in vivo. (a) The growth curves of tumors in nude mice (five mice/group) treated, respectively, by NS, control shRNA and TRIM27 shRNA plasmids. (b) Representative image of isolated tumors. (c) Tumor volume in the shTRIM27 group $\left(3.764 \pm 1.161 \mathrm{~mm}^{3}\right)$ was markedly lower than that in the shControl $\left(7.582 \pm 0.9224 \mathrm{~mm}^{3}\right)$ and NS $\left(8.922 \pm 1.323 \mathrm{~mm}^{3}\right)$ groups. (d) Tumor weight in the shTRIM27 group $(1.852 \pm 0.557 \mathrm{~g})$ was significantly lower than that in the shControl $(3.385 \pm 0.273 \mathrm{~g})$ and the NS $(3.925 \pm 0.678 \mathrm{~g})$ groups. (e) Western blotting assay showed that the tumors injected with TRIM27 shRNA expressed lower levels of TRIM27 compared with the shControl and NS groups. ${ }^{*} P<0.05$.

\section{DISCUSSION}

TRIM27, which acts as the member of TRIM protein family, could participate in a broad range of biological processes, including tumor development and metastasis. ${ }^{3}$ It has been reported that TRIM27 is highly expressed in endometrial endometrioid adenocarcinoma tissues compared with normal endometrium, and there is no significant association between TRIM27 expression and any of the clinicopathological parameters tested: age, FIGO stage, tumor grade, myometrium invasion, and pelvic lymph node metastasis. ${ }^{13,14}$ Iwakoshi et $a l^{15}$ reported that TRIM27 expression was detected in the nuclei of lung cancer cells but not in non-malignant cells, and there was no significant relationship between TRIM27 expression and any of the factors, except for thyroid transcription factor 1 (TTF-1) expression. Tezel et al ${ }^{16}$ reported that TRIM27 expression was observed in $41.4 \%$ of breast carcinomas but not in 10 benign breast tissues, and TRIM27 expression correlated with epidermal growth factor receptor II (ERBB2) protein expression and ERBB2 gene amplification in breast cancer. Furthermore, Tezel et al ${ }^{16}$ found a significant association between TRIM27 expression and age; however, there were no statistically significant correlations of TRIM27 expression with tumor size, lymph node status, estrogen receptor, progesterone receptor and 

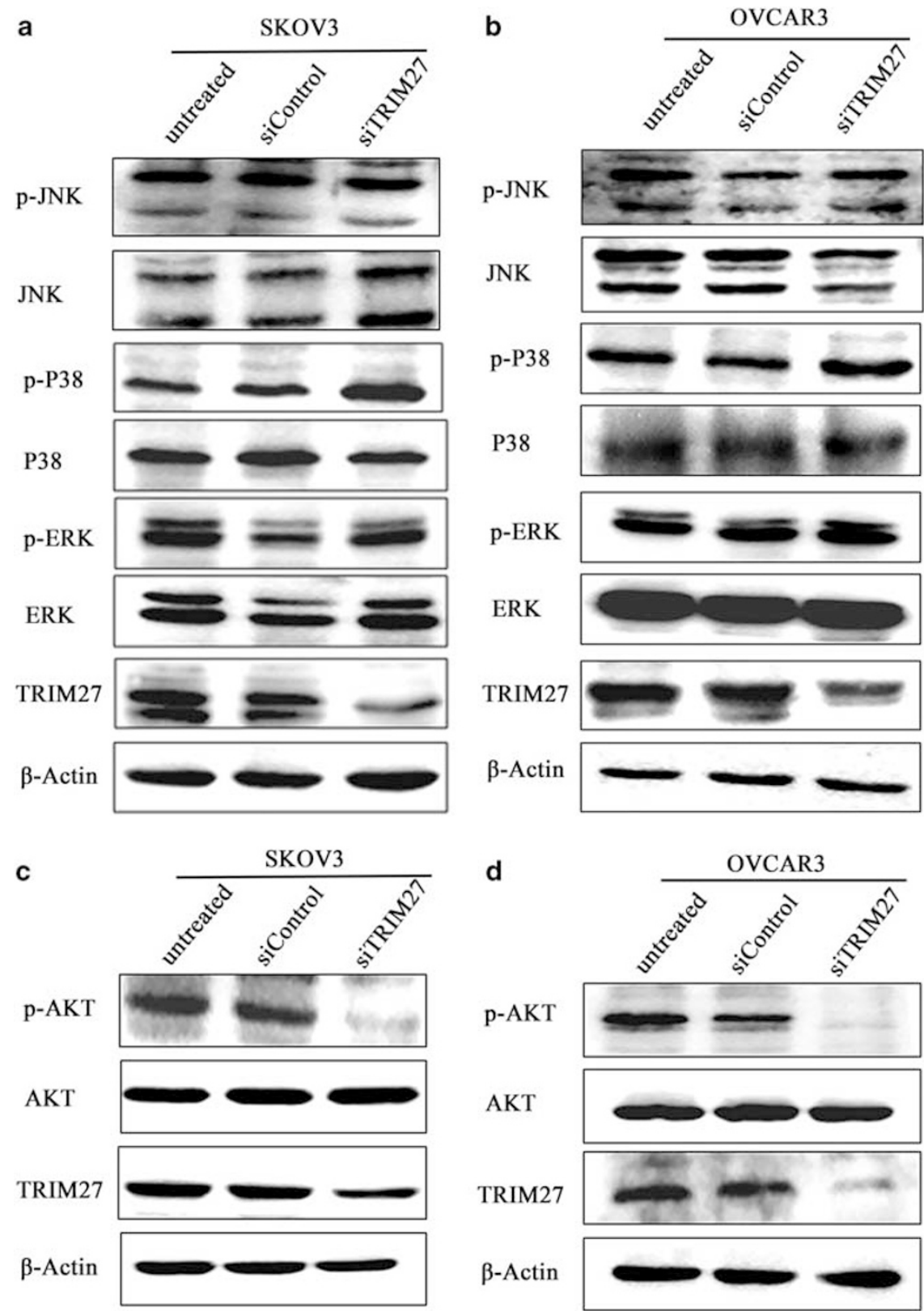

Figure 6 TRIM27 played important roles by affecting cell proliferation and apoptosis-related pathways. (a and b) Western blotting assay showed that the expression of p-P38 in the SKOV3 and OVCAR3 cells transfected with TRIM27-specific siRNA was increased compared with the control groups. (c and $\mathbf{d}$ ) Western blotting assay showed that the expression of p-AKT in the SKOV3 and OVCAR3 cells transfected with TRIM27-specific siRNA was decreased compared with the control groups.

grade. The above results showed that TRIM27 expression was upregulated in various tumor tissues, but there were differences in the associations of TRIM27 expression with clinicopathological characteristics of different cancer patients. This suggests that TRIM27 could be involved in the development and metastasis of some tumors, while the effective mechanism of TRIM27 may be tissue specific.

To elucidate the expression and clinical significance of TRIM27 in ovarian cancer, we evaluated the expression of TRIM27 protein in three human ovarian serous carcinoma-derived cells, SKOV3, OVCAR3 and CAOV3, and one human ovarian mucinous carcinoma-derived cell line, $3 \mathrm{AO}$, as well as 50 ovarian serous carcinomas, 26 normal ovarian and fallopian tube tissues. We also analyzed the association of TRIM27 expression with clinical and pathological parameters. We found that TRIM27 protein was mainly located in the cytoplasma of ovarian serous carcinoma cells and normal ovarian and fallopian tube epithelial cells. Moreover, TRIM27 expression was higher in ovarian serous carcinoma cells than that in normal ovarian and fallopian 
tube epithelial cells, and high expression of TRIM27 was significantly related to FIGO stage of ovarian serous carcinoma patients. Our results also showed that the ovarian carcinomas expressing higher levels of TRIM27 were easy to have metastasis. Among patients with metastasis, most of them had fallopian tube, uterus, omentum, intestinal wall or appendix involvement, and few of them had lymph node or distant metastasis. These data indicate that TRIM27 expression could participate in the progression of ovarian cancer.

When our manuscript was being prepared, another study on TRIM27 in ovarian cancer by Horio et al ${ }^{17}$ was published. They detected the expression of TRIM27 in different histological types, including serous, mucinous, endometrioid carcinomas and clear cell carcinoma, by IHC and analyzed the associations of TRIM27 expression with clinicopathological factors of these patients. However, there were some differences between their results and ours. Their results showed that strong TRIM27 expression was specifically detected in the nuclei of serous, mucinous, endometrioid carcinomas and clear cell carcinoma but not in those of nonmalignant cells surrounding the cancer cells. We speculate that TRIM27 may shuttle between the cytoplasma and nucleus, which induces different subcellular distributions of TRIM27. In addition, they observed that no significant association was found between TRIM27 expression and any clinicopathological parameters, including age, FIGO stage and histological type. This may be because we only analyzed the association of TRIM27 expression with clinical and pathological parameters of ovarian serous carcinoma patients, while they analyzed the relationship between TRIM27 expression and clinical and pathological parameters of different histological types of ovarian cancers.

At present, the mechanism by which TRIM27 expression contributed to the clinical outcome of cancer patients remains unclear. Tsukamoto et al ${ }^{13}$ reported that TRIM27 knockdown affected the proliferation of human cervical adenocarcinoma cell line HeLa cells but not of the other two cell lines (human endometrial adenocarcinoma cell line HE50 and human breast adenocarcinoma cell line MDA-MB-231 cells). These findings further suggest that TRIM27 may have cell-type- or tissue-specific function and TRIM27 may regulate the expression of different genes involved in cell proliferation. To further explore the role of TRIM27 in ovarian carcinogenesis, we investigated the effect of TRIM27 knockdown on the proliferation of ovarian cancer cells in vitro and in vivo. TRIM27 knockdown not only inhibited the proliferation and colonyforming ability of SKOV3 and OVCAR3 cells in vitro but also suppressed the growth of SKOV3 cells in nude mice. Our results are not in accordance with the report of Horio et al. ${ }^{17}$ They found that TRIM27 knockdown did not affect the proliferation rate of ovarian cancer cell lines in vitro, while shRFP-expressing cells exhibited a significant decrease in tumor growth in an athymic mouse xenograft model compared with shControl-expressing cells. The reason of this discrepancy needs to be further investigated.
Next we evaluated the effect of TRIM27 knockdown on cell cycle and early apoptosis of ovarian cancer cells. Our results showed that TRIM27 knockdown increased cell number in S-phase in SKOV3 cells, suggesting that downregulation of TRIM27 expression induced the S-phase arrest of ovarian cancer cells. ATR and Chk1 are required for the S-phase checkpoint. Chk1 is regulated by ATR through phosphorylation, forming the ATR-Chk1 pathway. It has been reported that the activation of Chk1 could phosphorylate a variety of substrate proteins, resulting in cell cycle arrest. ${ }^{18}$ Therefore, we detected the expression of ATR, p-ATR, Chk1 and p-Chk1 and found that downregulation of TRIM27 expression increased the expression of p-ATR and p-Chk1, which suggests that TRIM27 downregulation could activate S-phase checkpoint kinases and induce S-phase arrest. In addition, the results of Annexin V/PI double dyeing showed that reduced TRIM27 expression in SKOV3 cells resulted in significantly higher apoptosis. Therefore, we speculate that TRIM27 may regulate cell proliferation by affecting the cell cycle and apoptosis of ovarian cancer cells. Similarly, Horio et al ${ }^{17}$ reported that RFP depletion by siRNA sensitized the ovarian cancer cell lines to carboplatin and paclitaxel through an increase in apoptosis. However, So Dho and Kwon ${ }^{19}$ reported that overexpression of TRIM27 induced apoptosis of human embryonic kidney 293 cells by two distinct cell death pathways: one via caspase pathway independent of mitochondrial events, and one via stress-activated MAP kinase pathway that involves JNK and P38 kinase. This may be because different cell types were studied.

TRIM27 is a fusion partner with the RET tyrosine kinase ${ }^{5}$; the catalytic activity level of the hybrid TRIM27-RET protein is higher than that of RET alone. Expression of the fusion proteins is thought to result in ligand-independent kinase activation and subsequent cell proliferation and tumorigenesis. As RET participates in cell proliferation and differentiation via the Ras-MAPK and PI3K-AKT pathways, we speculate that TRIM27 take effects by the same pathways. ${ }^{20,21}$ We next detected the effect of TRIM27 knockdown on the activation of MAPK pathway (ERK1/2, p-ERK1/2, SAPK/JNK, p-SAPK/JNK, P38 MAPK, p-P38 $\mathrm{MAPK}$ ) and PI3K-AKT (AKT and p-AKT) pathways. The results showed that TRIM27 knockdown increased the expression of p-P38 and decreased the expression of p-AKT, whereas it had no effect on the expression of p-ERK and p-JNK. It has been reported that the p38MAPK pathway could participate in a variety of physiological and pathological processes, including cell proliferation, cell cycle regulation and cell apoptosis. ${ }^{22,23}$ Palanivel et al ${ }^{24}$ found that Verrucarin A (VA) induces apoptosis and cell cycle deregulation in MCF-7 cells through ROS-dependent p38MAPK activation. Kim et $a^{25}$ also reported that the activation of the p38MAPK pathway induced Bax phosphorylation and mitochondrial translocation and apoptosis of HepG2 cells. In addition, PI3K/AKT signaling pathways have important roles in regulating cell proliferation, 
cycle and apoptosis by inducing the activation of major downstream effectors, such as Bad, caspase 9, IKK $\alpha$, GSK-3, FKHR, p21 and p27.26,27 AKT activation could enhance resistance to apoptosis and induce cell survival signaling through multiple downstream pathways. ${ }^{28-31}$ Combined with these results, we speculate that TRIM27 knockdown could promote the activation of the p38MAPK pathway and suppress the activation of the PI3K/AKT pathway and then inhibit cell proliferation and induce cell apoptosis and cell cycle arrest. However, the exact effect mechanism of TRIM27 in ovarian carcinogenesis needs to be explored in the future.

In conclusion, we demonstrated that TRIM27 expression was significantly increased in ovarian serous carcinoma cells and correlated positively to metastasis and FIGO stage of patients with ovarian serous carcinoma. TRIM27 knockdown could inhibit cell proliferation and colony-forming ability and induce cell cycle arrest and apoptosis of ovarian cancer cells by upregulating the expression of p-P38 and downregulating the expression of p-AKT. These results suggest that TRIM27 could be involved in tumor development and may be a new molecular target for diagnosis and treatment of ovarian cancer.

\section{ACKNOWLEDGMENTS}

This work was supported by the National Natural Science Foundation of China (81471437, 81172863), Natural Science Foundation of Shandong (ZR2012HM091, ZR2013HM105), Independent Innovation Foundation of Shandong University (2012ZD045), Postdoctoral Innovation Program of Shandong Province (201102015) and China Postdoctoral Science Foundation funded project (2012M511516).

\section{DISCLOSURE/CONFLICT OF INTEREST}

The authors declare no conflict of interest.

1. Siegel R, Naishadham D, Jemal A. Cancer Statistics, 2012. CA Cancer J Clin 2012;62:10-29.

2. Kajiyama $H$, Shibata $K$, Mizuno $M$ et al. Long-term survival of young women receiving fertility-sparing surgery for ovarian cancer in comparison with those undergoing radical surgery. $\mathrm{Br} \mathrm{J}$ Cancer 2011;105:1288-1294.

3. Cao T, Borden KL, Freemont PS et al. Involvement of the Rfp tripartite motif in protein-protein interactions and subcellular distribution. J Cell Sci 1997:110(Pt 14):1563-1571.

4. Cao T, Shannon M, Handel MA et al. Mouse Ret finger protein (Rfp) proto-oncogene is expressed at specific stages of mouse spermatogenesis. Dev Genet 1996;19:309-320.

5. Takahashi M, Inaguma $\mathrm{Y}$, Hiai $\mathrm{H}$ et al. Developmentally regulated expression of a human "finger"-containing gene encoded by the 5 ' half of the ret transforming gene. Mol Cell Biol 1988;8:1853-1856.

6. Shimono $\mathrm{Y}$, Murakami $\mathrm{H}$, Kawai $\mathrm{K}$ et al. Mi-2 beta associates with $\mathrm{Brg} 1$ and Ret finger protein at the distinct regions with transcriptional activating and repressing abilities. J Biol Chem 2003;278:51638-51645.

7. Tezel G, Nagasaka $T$, Iwahashi $\mathrm{N}$ et al. Different nuclear/cytoplasmic distributions of Ret finger protein in different cell types. Pathol Int 1999;49:881-886.

8. Tezel G, Nagasaka T, Shimono $Y$ et al. Differential expression of Ret finger protein in testicular germ cell tumors. Pathol Int 2002;52:623-627.

9. Hatakeyama S. Trim proteins and cancer. Nat Rev Cancer 2011;11: 792-804.
10. Zoumpoulidou G, Broceno C, Li H et al. Role of the tripartite motif protein 27 in cancer development. J Natl Cancer Inst 2012;104: 941-952.

11. Cao $\mathrm{T}$, Duprez $\mathrm{E}$, Borden $\mathrm{KL}$ et al. Ret finger protein is a normal component of Pml nuclear bodies and interacts directly with Pml. J Cell Sci 1998;111(Pt 10):1319-1329.

12. Townson SM, Kang K, Lee AV et al. Novel role of the Ret finger protein in estrogen receptor-mediated transcription in Mcf-7 cells. Biochem Biophys Res Commun 2006;349:540-548.

13. Tsukamoto $\mathrm{H}$, Kato $\mathrm{T}$, Enomoto $\mathrm{A}$ et al. Expression of Ret finger protein correlates with outcomes in endometrial cancer. Cancer Sci 2009;100: 1895-1901.

14. Tezel GG, Ordulu Z, Himmetoglu C et al. The selective expression of Ret finger protein in endometrial cancer: can Rfp be a marker of serous carcinomas? Turk Patoloji Derg 2012;28:213-219.

15. Iwakoshi A, Murakumo $Y$, Kato $T$ et al. Ret finger protein expression is associated with prognosis in lung cancer with epidermal growth factor receptor mutations. Pathol Int 2012;62:324-330.

16. Tezel GC, Uner A, Yildiz I et al. RET finger protein expression in invasive breast carcinoma: relationship between RFP and ErbB2 expression. Pathol Res pract 2009;205:403-408.

17. Horio $M$, Kato $T, M i i \mathrm{~S}$ et al. Expression of RET finger protein predicts chemoresistance in epithelial ovarian cancer. Cancer Med 2012;1: 218-229.

18. Mallikarjun $P$, Navjotsingh $P$, Zheng D. Checkpoint kinase 1 in DNA damage response and cell cycle regulation. Cell Mol Life Sci 2013; 70: 4009-4021.

19. Dho SH, Kwon KS. The Ret finger protein induces apoptosis via its RING finger-B box-coiled-coil motif. J Biol Chem 2003;278: 31902-31908.

20. Kodama Y, Asai N, Kawai K et al. The RET proto-oncogene: a molecular therapeutic target in thyroid cancer. Cancer Sci 2005;96:143-148.

21. Ichihara M, Murakumo Y, Takahashi M. RET and neuroendocrine tumors. Cancer Lett 2004;204:197-211.

22. Ashraf $\mathrm{Ml}$, Ebner $\mathrm{M}$, Wallner $\mathrm{C}$ et al. A p38MAPK/MK2 signaling pathway leading to redox stress, cell death and ischemia/ reperfusion injury. Cell Commun Signal 2014;12:6.

23. Kandil S, Brennan L, McBean GJ. Glutathione depletion causes a JNK and p38MAPK-mediated increase in expression of cystathioninegamma-lyase and upregulation of the transsulfuration pathway in C6 glioma cells. Neurochem Int 2010;56:611-619.

24. Palanivel K, Kanimozhi V, Kadalmani B. Verrucarin A alters cell-cycle regulatory proteins and induces apoptosis through reactive oxygen species-dependent p38MAPK activation in the human breast cancer cell line MCF-7. Tumour Biol 2014;35:10159-10167.

25. Kim BJ, Ryu SW, Song BJ. JNK- and p38 kinase-mediated phosphorylation of Bax leads to its activation and mitochondrial translocation and to apoptosis of human hepatoma HepG2 cells. J Biol Chem 2006;281: 21256-21265.

26. Matsuoka T, Yashiro M. The role of PI3K/Akt/mTOR signaling in gastric carcinoma. Cancers 2014;6:1441-1463.

27. Xing $X$, Wang J, Xing LX et al. Involvement of MAPK and PI3K signaling pathway in sterigmatocystin-induced G2 phase arrest in human gastric epithelium cells. Mol Nutr Food Res 2011;55:749-760.

28. Xu GP, Zhao W, Zhuang JP et al. Matrine inhibits the growth and induces apoptosis of osteosarcoma cells in vitro by inactivating the Akt pathway. Tumour Biol 2014;36:1653-1659.

29. Wang J, Chu ES, Chen HY et al. microRNA-29b prevents liver fibrosis by attenuating hepatic stellate cell activation and inducing apoptosis through targeting PI3K/AKT pathway. Oncotarget 2014;6: 7325-7338.

30. Zhao GX, XU LH, Pan $\mathrm{H}$ et al. The BH3-mimetic gossypol and noncytotoxic doses of valproic acid induce apoptosis by suppressing cyclin-A2/Akt/FOXO3a signaling. Oncotarget; advance online publication, 16 October 2015 [e-pub ahead of print].

31. Wang KF, Yang H, Jiang WQ et al. Puquitinib mesylate (XC-302) induces autophagy via inhibiting the PI3K/AKT/mTOR signaling pathway in nasopharyngeal cancer cells. Int J Mol Med; advance online publication, 16 October 2015 [e-pub ahead of print]. 